\title{
The New Iron Age
}

\section{Citation}

Xu, Cenke and Subir Sachdev. 2008. The new iron age. Nature Physics 4, no. 12: 898-900.

\section{Published Version}

doi:10.1038/nphys 1137

\section{Permanent link}

http://nrs.harvard.edu/urn-3:HUL.InstRepos:11190321

\section{Terms of Use}

This article was downloaded from Harvard University's DASH repository, and is made available under the terms and conditions applicable to Open Access Policy Articles, as set forth at http:// nrs.harvard.edu/urn-3:HUL.InstRepos:dash.current.terms-of-use\#OAP

\section{Share Your Story}

The Harvard community has made this article openly available.

Please share how this access benefits you. Submit a story.

\section{Accessibility}




\section{The new iron age}

\section{CENKE XU and SUBIR SACHDEV*}

are in the Department of Physics, Harvard University, Cambridge MA 02138, USA

*e-mail: subir_sachdev@harvard.edu

The discovery of a new class of high-temperature superconductors based on iron tests the limits of current theoretical and computational tools for the understanding of strongly correlated systems.

If you happened recently to run into a sleep-deprived condensed-matter physicist at the local coffee shop, and asked her what's been keeping her awake at night, she'd probably tell you with excitement that she and her colleagues have been working around the clock on a new class of high-temperature superconductors. These new superconductors are iron-based, in contrast to the so-called cuprates — the 'traditional' high-temperature superconductors based on copper, which have been studied intensively during the past two decades since the discovery of their superconducting properties. The cuprates have been the prototypes of strongly correlated electron systems and the impetus for remarkable developments in the theory thereof, yielding insight into new phases and critical points that have been found by experiment in such systems. However, many experimentally relevant problems have remained theoretically intractable, especially those concerning the transition to temperatures above the superconducting critical temperature $\left(T_{\mathrm{c}}\right)$ at low carrier concentrations. Now theorists have an opportunity to test their mettle on the new, iron-based superconductors. 
So far, there are three groups of materials in this new superconductor family, all of which display superconducting behaviour upon applying external pressure or introducing certain type of dopants. First there is the so-called 1111 group, which encompasses materials with composition $\mathrm{MFeAsO}$, where $\mathrm{M}$ represents a lanthanide such as $\mathrm{La}, \mathrm{Ce}, \mathrm{Pr}, \mathrm{Sm}$ or Eu. These compounds have been found to be superconducting, with a transition temperature as high as $55 \mathrm{~K}$ (ref. 1,2), when doped with electrons by replacing oxygen with fluoride, or by simply reducing the oxygen content. The second group, dubbed ' 122 ', has the composition $\mathrm{MFe}_{2} \mathrm{As}_{2}$, where $\mathrm{M}$ is an alkaline-earth element. To make them superconducting, the 122 compounds are usually doped with holes, by replacing alkaline earths with alkali elements ${ }^{3}$. The third group consists of only Fe and one of the chalcogen elements. An example is FeSe, which has a transition temperature of about $8 \mathrm{~K}$ in the absence of externally applied pressure or doping. However, when subject to doping or high pressure, the transition temperature of FeSe can be enhanced ${ }^{4}$ up to $27 \mathrm{~K}$. This third family may be expected to gain most attention, in part because of its relatively simple structure, but also because of the inherently poisonous nature of arsenides.

Already at first glance it seems that the iron-based superconductors are more complicated than the cuprates, and that a theoretical description of their properties will be challenging. Consider, for instance, the copper ion $\mathrm{Cu}^{2+}$. It has only one, unfilled $d$-orbital. This suggests that a single narrow-band model containing both kinetic energy and on-site repulsive coulomb interactions — such as the Hubbard model or its low-energy descendent the 't-J' model — should be able to describe all the major properties of the cuprates. In contrast, the ion $\mathrm{Fe}^{2+}$ has six electrons in five $d$-orbitals, and their filling depends on the detailed competition between the Hund's 
coupling and the 'crystal field' splitting, the lattice-potential-induced energy splitting between the $d$-orbitals. Thus, a multi-band approach is likely to be needed. This has indeed been seen in comparisons between a multi-band analogue of the Hubbard model used to describe the cuprates and calculations using the local-density approximation (LDA) to density-functional theory — a benchmark computational method for band-structure calculations. Numerical results obtained from LDA-based computations have so far agreed qualitatively with results ${ }^{5}$ obtained from Fermi surface characterization using angle-resolved photoemission spectroscopy (ARPES). To correspondingly reproduce the correct topology and the wave function components at the Fermi level using a multi-band analogue to the Hubbard model, at least four $d$ bands are needed ${ }^{6}$. Consequently, for an accurate description of the iron-based superconductors, we have to move from a 'simple' one-band Hubbard model to at least a four-band Hubbard-like model.

A central question, however, is whether the LDA-like physics of weakly interacting electron bands provides a suitable starting point for understanding the phenomenology of the iron-based superconductors, or whether the local correlations have to be taken carefully into account in an extended Hubbard model. In the cuprates, the undoped parent compounds are Mott insulators - carriers are localized due to strong on-site coulomb repulsions. This is naturally accounted for in the Hubbard model description, and is beyond the capability of LDA. In addition, doped Hubbard models seem a fairly convincing route towards a mechanism for high-temperature superconductivity, and the variety of competing spin and charge orders that have been observed in the doped cuprates. On the other hand, the recent claims ${ }^{7}$ of electron pockets in holedoped cuprates in a $60 \mathrm{~T}$ magnetic field, if proved correct, suggest that correlations 
may not be as strong as previously thought: such electron pockets are more naturally described in a theory of metallic electron bands ${ }^{8}$ in the presence of magnetic order induced by the field ${ }^{9}$. Thus it may well be that the correlations in the Mott insulator are crucial in inducing the competing spin order, but that the Fermi surface configurations are amenable to a LDA-like theory of electron bands in the presence of this order.

In contrast to the cuprates, in the iron-based superconductors the undoped parent compounds are not Mott insulators, thus suggesting that correlations are not as strong as in the cuprates. However, magnetic order has been observed ${ }^{10}$, as we will discuss below, and can easily be understood in the context of a strong-correlation Hubbard model. Also, the traditional BCS theory based on electron-phonon coupling is believed to be incapable of inducing such a high transition temperature in these compounds ${ }^{11}$, therefore the coulomb interactions should be carefully taken into account.

Based on all of these observations the most likely scenario is that, for the iron-based superconductors, we are in an intermediate coupling regime - one in which kinetic and interaction energies are comparable - where computations are most difficult and simple physical pictures are likely to be inadequate. As now seems to be the case for the cuprates, it is probable that the data from different experimental probes will require complementary theories using weak- or strong-coupling strategies to unravel the physics. Adding to these considerations is the complexity related to the multi-band approach that is needed for an accurate microscopic description of the physics of the 
iron-based superconductors. It is clear that we are faced with a problem that will stretch the limitations of our mathematical and computational tools.

But the difficulty of understanding the microscopic theory of the iron-based superconductors should not hinder us from understanding their phenomenology, which is anyway more directly relevant to future applications based on these materials. Let us for a moment go back to that condensed-matter physicist in the coffee shop. After she has told you about the new superconductors, the first question you might ask is, "What's the highest transition temperature?" After getting a satisfactory answer, you might follow up with "What is the pairing symmetry of these superconductors?" The chances are that this condensed-matter physicist will stop being excited and answer quite awkwardly that, after nearly a year of hard work, we still do not know for sure. At present, the answer seems to be sample- as well as probe-dependent.

During the first few months of research in this field, the evidence favoured $d$-wave pairing, which is characterized by the phase of the Cooper-pair wavefunction changing sign four times upon circling the Fermi surface. Evidence for this came mainly from measurements that probed the low-energy part of the density of states below $\mathrm{T}_{\mathrm{c}}$. For instance, measurements of both the NMR relaxation rate and the specific heat suggested the existence of nodal lines with gapless quasiparticles at certain points on the Fermi surface ${ }^{12}$. This interpretation was subsequently supported by scanning tunnelling microscopy (STM) and Andreev-reflection measurements on polycrystalline samples ${ }^{13,14}$. The STM data revealed a linear-in-energy dependence of the local density-of-states below $\mathrm{T}_{\mathrm{c}}$; in turn, measurements of Andreev reflection 
displayed a zero-bias peak, believed to be due to an Andreev bound state at the surface. From these results it was inferred that ordinary $s$-wave symmetry could be excluded. Just when it seemed that a consensus was emerging, ARPES measurements revealed a fully gapped Fermi surface, without any sign of nodal lines, this time in high-quality, single-crystal samples of the ' 122 ' compounds ${ }^{15}$. Challenged by these discrepancies, iron-willed physicists have made various efforts to come up with a special pairing symmetry to reconcile all of the experimental facts. But so far, a perfect solution has not emerged.

Although measurements on different compounds have revealed discrepancies with regard to the pairing symmetry, the results do share one common signature: in the non-superconducting parent compound, a lattice distortion accompanied by a spin density wave (SDW) - a periodic spatial modulation of spins - occurs at wavevectors $(\pi, 0)$ or $(0, \pi)$ in the low temperature regime ${ }^{10}$ (represented by the blue and green lines in the phase diagram, Fig. 1). Both the lattice distortion and the SDW are suppressed and eventually destroyed by increased doping and/or pressure ${ }^{16}$ (Fig. 1). At these particular wavevectors, an accurate theoretical description of the phenomenology needs to take into account first- and second-nearest-neighbour antiferromagnetic exchange interactions [fine] between the Fe ions ${ }^{17}$ in the FeAs layer.

Interestingly, there seems to be a difference in the characteristics [fine] of these signatures between the ' 1111 ' and ' 122 ' families of the iron-based superconductors. In the 1111 compounds, the lattice distortion and the SDW occur at two different transition temperatures ${ }^{18}$, but still quite close together in the phase diagram. In the 122 
compounds, on the other hand, the two signatures occur at the same transition temperature, accompanied by a finite jump in crystal structure's lattice constants ${ }^{19,20}$. To account for the different behaviour between the 1111 and 122 compounds, we and other authors have proposed a unified theory of the lattice distortion and SDW, which attributes the lattice distortion to an Ising order parameter defined purely magnetically ${ }^{21,22}$. Because the lattice distortion and SDW are strongly correlated, if they occur at the same transition temperature it implies a first-order nature for the phase .This unified understanding of magnetism and lattice distortion is based only on the symmetry of the system, and is hence independent of the microscopic model.

However, other mysteries remain. As we mentioned before, the $\mathrm{Fe}^{2+}$ ion has 6 electrons in the $3 d$ orbitals, which means that the total spin of one Fe ion is either 1 or 2. However, the SDW magnetic moment in 1111 samples is only about $0.3 \mathrm{Bohr}$ magnetons ${ }^{10}$. Clearly the magnetism cannot be fully understood in a strong correlation model, and the challenge remains to understand the consequences of the metallic electrons.

Many other interesting questions about these compounds have been raised, and efforts have been made to answer them. For instance, do the spin density wave and superconductivity compete strongly with each other, or can they coexist? Is there a pseudogap phase above the superconducting state of these materials, as there is in the cuprates? Exploring all of these mysteries surrounding the iron-based superconductors will help in understanding the interplay between kinetic and interaction energy in condensed-matter physics in general, and perhaps lead us into a new iron age. 


\section{REFERENCES}

1. Ren, Z.-A. et al. Europhys. Lett. 83, 17002 (2008).

2. Chen, X.^H., Wu, T., Wu, G., Liu, R.^H., Chen, H. \& Fang, D.^F. Nature 453, 761-762 (2008).

3. Sasmal, K. et al. Phys. Rev. Lett. 101, 107007 (2008).

4. Mizuguchi, Y., Tomioka, F., Tsuda, S., Yamaguchi, T. \& Takano, Y. Appl. Phys. Lett. 93, 152505 (2008).

5. Lu, D.^H. et al. arXiv:0807.2009 (2008).

6. Lee, P.^A. \& Wen, X.-G. arXiv:0804.1739 (2008).

7. LeBoeuf, D. et al. Nature 450, 533-536 (2007).

8. Chubukov, A.^V. \& Morr, D.^K. Physics Reports 288, 355-387 (1997).

9. Demler, E., Sachdev, S. \& Zhang, Y. Phys. Rev. Lett. 87, 067202 (2001).

10. de la Cruz, C. et al. Nature 453, 899-902 (2008).

11. Boeri, L., Dolgov, O.^V. \& Golubov, A.^A. Phys. Rev. Lett. 101, 026403 (2008)

12. Nakai, Y., Ishida, K., Kamihara, Y., Hirano, M. \& Hosono, H. arXiv:0804.4765 (2008).

13. Wang, Y., Shan, L., Fang, L., Cheng, P., Ren, C. \& Wen, H.-H. arXiv:0806.1986 (2008).

14. Millo, O. et al. Phys. Rev. B 78, 092505 (2008).

15. Ding, H. et al. Europhys. Lett. 83, 47001 (2008).

16. Zhao, J. et al. arXiv:0806.2528 (2008).

17. Si, Q. \& Abrahams, E. Phys. Rev. Lett. 101, 076401 (2008).

18. McGuire, M.^A. et al. Phys. Rev. B 78, 094517 (2008). 
19. Krellner, C., Caroca-Canales, N., Jesche, A., Rosner, H., Ormeci, A. \& Geibel, C. Phys. Rev. B 78, 100504(R) (2008).

20. Zhao, J. et al. Phys. Rev. B 78, 140504 (2008).

21. Xu, C., Müller, M. \& Sachdev, S. Phys. Rev. B 78, 020501(R) (2008).

22. Fang, C., Yao, H., Tsai, W.-F., Hu, JP \& Kivelson, S.^A. Phys. Rev. B 77, 224509 (2008).

23. Kreyssig, A. et al. arXiv:0807.3032 (2008).

Figure 1

A proposed phase diagram for the iron-based superconductors as a function of doping $(\mathrm{x})$, temperature $(\mathrm{T})$, and pressure $(\mathrm{P})$, reflecting phase transitions observed in recent experimental works. The orange lines delimit the region in phase diagram where the new iron-based compounds are superconducting. The blue and green lines signify second-order phase transitions characterized by a lattice distortion and spin-density wave $(S D W)$, respectively. In a certain pressure regime, the two transitions occur simultaneously, characterized by the thick red line, in which case the transition is of first order. The dashed line indicates a volume-collapse transition found ${ }^{23}$ in $\mathrm{CaFe}_{2} \mathrm{As}_{2}$, which, we believe, may be indicative of strong correlation effects. 